\title{
Unresolved or Contradictory Issues About Management of Patients With Patent Foramen Ovale and Previous Cryptogenic Stroke: Additional Randomized Controlled Trials Are Eagerly Awaited
}

\author{
Renato De Vecchis ${ }^{\mathrm{a}, \mathrm{c}}$, Cesare Baldi ${ }^{\mathrm{b}}$
}

\begin{abstract}
Stating a well-codified and widely accepted therapeutic conduct for patients with patent foramen ovale (PFO) and previous cryptogenic stroke is made difficult and somewhat controversial by several issues remained unresolved so far. In this short review, some aspects of the possible role played by the PFO in the pathogenesis of cryptogenic stroke are succinctly analyzed. First, some aspects of cardiovascular anatomy of the human fetus and the adult are outlined. Subsequently, the three randomized controlled trials (RCTs) that have been accomplished so far to compare the implant of a transeptal occluding device with a simple medical therapy in patients with PFO and history of cryptogenic stroke are briefly examined. These RCTs, when assessed using the "intention to treat" method, do not show a greater protective effect of therapy with transeptal device as regards the recurrences of stroke. Afterwards, there is a brief presentation of the findings of several meta-analyses that have been derived from the three above mentioned RCTs, whose results are strikingly discordant with each other. In fact, some of them come to the conclusion that the transcatheter closure of PFO does not offer significant advantages compared to antithrombotic therapy for the secondary prevention of cryptogenic stroke, while others based on subgroup analyses argue that the transcatheter closure of PFO with Amplatzer device, differently from the one performed using the STARFlex device, would be associated with significantly lower incidence of cerebrovascular events compared with medical therapy alone. Finally, the authors argue the need to adhere to the current scientific guidelines. They substantially deny an alleged superior efficacy of transcatheter PFO occlusion compared to medical therapy with antithrombotic agents (anticoagulants or antiplatelet agents), except for selected cases of patients with documented PFO and concomitant clinical-instrumental picture of deep venous
\end{abstract}

Manuscript accepted for publication February 15, 2016

aCardiology Unit, Presidio Sanitario Intermedio "Elena d'Aosta“, ASL Napoli 1 Centro, Napoli, Italy

bHeart Department, Interventional Cardiology, A.O.U. "San Giovanni di Dio e Ruggi d'Aragona", Salerno, Italy

${ }^{\mathrm{c} C o r r e s p o n d i n g ~ A u t h o r: ~ R e n a t o ~ D e ~ V e c c h i s, ~ C a r d i o l o g y ~ U n i t, ~ P r e s i d i o ~ S a n i-~}$ tario Intermedio "Elena d'Aosta", ASL Napoli 1 Centro, Napoli, Italy.

Email: devecchis.erre@virgilio.it

doi: http://dx.doi.org/10.14740/jocmr2491w thrombosis.

Keywords: Patent foramen ovale; Cryptogenic stroke; Secondary prevention

\section{Introduction}

The issue of the real efficacy of transcatheter closure of the patent foramen ovale (PFO) for secondary prophylaxis of stroke or transient ischemic attack (TIA) is periodically revisited by means of expert opinions and editorials [1-3] propitiated or elicited by recurrent meta-analyses, sometimes conflicting each other [4-18], and built on the basis of the only three randomized controlled trials (RCTs) [19-21] published so far on this topic. All of the three trials addressed the issue of whether, in patients with a history of cryptogenic stroke or TIA, transcatheter closure of PFO offers a real advantage in terms of survival free from relapses of stroke or TIA, when compared with medical therapy consisting of anticoagulants and/or antiplatelet agents. On the whole, all three trials are concordant in ruling out that PFO closure by septal occluder device, compared with medical therapy, is able to yield better outcomes (composite of all-cause death, neurologic-cause death, nonfatal stroke, or TIA in the CLOSURE I trial [19]; composite of death, non-fatal stroke, TIA, or peripheral embolism in the PC trial [20]; composite of all-cause death or ischemic stroke, fatal or non-fatal, in the RESPECT trial [21]). By contrast, the numerous meta-analyses conducted by aggregating the study data, although almost all were built on the basis of only three RCTs made so far, are discordant with each other. Indeed, some investigators have interpreted the RCT data by excluding a benefit from the PFO closure $[9,10,13$, $14,17,18]$, while others have asserted that an advantage, albeit modest, is apparent for the interventional approach based on negative overall results, but positive findings in an "as treated" population or subgroup analysis $[4,5,7]$. Moreover, several researchers have argued that there were insufficient data to support benefit or harm $[6,8]$, while someone has provided a diametrically opposed interpretation, by underlining the positive results of the closure made using the Amplatzer device 
$[15,16]$ or by arguing that a clear benefit is evident with the use of the interventional strategy $[11,12]$.

In this review, we will analyze some of these issues, in the consciousness that it is, however, unrealistic to think that we can solve, with only a few considerations, the difficult questions posed on the carpet, covering aspects of pathology, pathophysiology, and clinical research methodology, as well as techniques for pooling and presentation of data in meta-analyses from studies in the literature.

\section{Brief Notions on Anatomy and Epidemiology Concerning the PFO}

The foramen ovale is formed towards the end of the fourth week of gestation. Initially, the atria are separated from each other by the "septum primum", except for a small discontinuity in the wall of the interatrial septum, called the ostium primum. As the septum primum grows, the ostium primum is reduced in size until it disappears [3, 22]. Before this is realized, the blood flow from the inferior vena cava slowly produces an excavation in the "septum primum", thus forming the "ostium secundum". The ostium secundum provides a communication between the atria after the ostium primum becomes completely unviable and closes. Subsequently, a second wall of tissue, the septum secundum, grows near to the ostium secundum in the right atrium. At this point, the blood flow passes exclusively from the right to the left atrium through a narrow passageway that has been created in the meantime in the septum secundum. This passageway is called the foramen ovale or Botallo's foramen. Normally, this discontinuity of the wall is obliterated spontaneously at the moment of birth $[3,22]$. In fact, when the lungs begin to function at birth, the pulmonary pressure decreases, and left atrial pressure exceeds that in the right atrium. This pushes the septum primum against the septum secundum, functionally closing the foramen ovale. Over time, the septa fuse together, leaving a remnant of the original foramen ovale, the "fossa ovalis" $[3,22]$. However, in about $25 \%$ of adults, the foramen ovale does not undergo complete closure, but remains patent [23] Moreover, some scholars postulate that in this portion of adults, by maintaining direct communication between the right- and left-sided circulation, the PFO would serve as a potential passageway for paradoxical embolization (cerebral as well as peripheral embolic events) $[24,25]$.

\section{Cryptogenic Stroke: Definition and Some Patho- physiological Issues}

In technical language, a stroke is termed "cryptogenic" when its etiology cannot be attributed to any specific cause after an extensive search for the most common causes, such as atherosclerosis of the intracranial vessels, lacunar damage from hypertension, or embolus derived from a thrombus located in the left atrium, the left ventricular apex, or at the level of an ulcerated plaque of the aortic arch. In all cases, in which the site of origin of the thrombus or the exact pathogenic mechanism of cerebral ischemia is not identifiable, it would appear appropri- ate to use the term "cryptogenic stroke". In this regard, there are also the helpful considerations made by the scholars who have explored this topic for years. According to Kistler and Furie [1], for example, it is possible that a significant portion of cryptogenic strokes are embolic in nature. Therefore, in the presence of cerebral ischemia of uncertain or unknown origin, it would be extremely important to make a careful assessment of the possible sources of arterial embolism (ulcerated carotid plaques, for example) as well as a thorough study of the left atrial appendage, by means of transesophageal echocardiography (TEE) if the patient suffers from permanent or paroxysmal atrial fibrillation. However, this should take place after other major causes of stroke have been excluded: large vessel atherothrombotic disease (15\%), small vessel lacunar stroke $(25 \%)$, and other mechanisms, such as dissection or arteritis $(3 \%)$. Therefore, before attributing the origin of cerebral ischemia to extracranial sources of emboli, an endocranial thrombosis should be convincingly excluded by determining that the stroke topology is not lacunar and that the parent vessels supplying the territory of the ischemic stroke are free of intrinsic atherosclerosis, dissection, or other causes of stenosis. In the context of the uncertainties about the origin and pathogenesis of a considerable proportion of so-called cryptogenic ischemic strokes, i.e., approximately $40 \%$ of all strokes [1], the question of the possible role played by PFO in the stroke's genesis has been extensively debated. In fact, the role of a small discontinuity of the interatrial septum (IAS) in causing the stroke remains controversial. The arguments used by some to deny a relationship between PFO and stroke include the consideration that the defect is present as an autoptic finding in over $25 \%$ of Caucasians. Therefore, according to this school of thought, an explanation should be given about the reasons for which, in most cases, this septal anomaly does not show signs or symptoms, so as to merely constitute an unsuspected autoptic finding in individuals who died from causes other than stroke. Moreover, the argument that the thrombotic material can originate inside the foramen ovale and then produce embolic dissemination in the arterial circulation appears a rather fanciful argument, not supported by a convincing pathophysiological rationale. In addition, the argument that the paradoxical embolism may result from thrombi located in the systemic venous circulation appears even more questionable, because the transport of thrombotic masses from the systemic venous bed into the arterial circulation would entail the existence of a pressure gradient from the right toward the left side of the IAS, while it is known that only in the case of severe pulmonary hypertension, the pressure in the right atrial chamber reaches or exceeds that in the left atrium. Thus there are some concerns about the putative role of PFO as a risk factor for cerebral embolism. In particular, there are considerable perplexities about the concept that, on some particular circumstances, such as during the Valsalva maneuver, which is reproduced by the act of defecating or by coughing, the pressure in the right atrium would be able to rise to generate a gradient capable of directing the blood stream from the right toward the left atrium. According to these criticisms, PFO would not, in and of itself, be sufficient to allow the blood flow to drag into the systemic circulation an embolus generated by a thrombus located in the right atrium, inside the systemic venous circulation or in the 
Table 1. Strengths and Limitations of Meta-Analyses of PFO Closure RCTs for Stroke Recurrence

\begin{tabular}{llllll}
\hline Study & RCTs only & $\begin{array}{l}\text { ITT subset } \\
\text { only }\end{array}$ & $\begin{array}{l}\text { Random effect } \\
\text { statistical model }\end{array}$ & $\begin{array}{l}\text { Analysis of potential } \\
\text { adverse events }\end{array}$ & Conclusion beneficial \\
\hline Ntaios et al [4] & Yes & No & No & Yes & No overall; yes with Amplatzer \\
\hline Pineda et al [5] & Yes & No & Yes & Yes & No overall; yes as treated \\
\hline Kitsios et al [6] & Yes & No & Yes & Yes & Inconclusive \\
\hline Riaz et al [7] & Yes & No & Yes overall; yes as treated \\
Hakeem et al [8] & Yes & No & Yes & Yes & Probably \\
Kwong et al [9] & Yes & Yes & Yes & Yes & No \\
Nagaraja et al [10] & Yes & Yes & Yes & Yes & No \\
Rengifo-Moreno et al [11] & Yes & No & No & Yes & Yes \\
Khan et al [12] & Yes & No & No & Yes & No \\
Spencer et al [13] & Yes & No & Yes & Yes & No \\
Wolfrum et al [14] & No & No & Yes & Yes & No overall; yes with Amplatzer \\
Capodanno et al [15] & No & Yes & Yes & No & No overall; yes with Amplatzer \\
\hline Pandit et al [16] & Yes & Yes & No & Yes & No \\
\hline Udell et al [17] & Yes & Yes & Yes & Yes & No \\
\hline Li et al [18] & Yes & Yes & & Yes & \\
\hline
\end{tabular}

ITT: intention-to-treat; RCT: randomized controlled trial.

PFO itself, the latter condition being hypothesized if this passageway is anfractuous and suitable for propitiating the local aggregation of platelets, especially in the presence of septal aneurysm.

\section{The Real Effectiveness of Transcatheter Clo- sure of PFO With Respect to Medical Therapy in the Secondary Prevention of Cryptogenic Stroke: Possible Interpretations of the Discrep- ancies Found by Examining the Results of the Available Meta-Analyses}

An important contribution to the study of the effectiveness of a PFO occlusion device for the prevention of recurrent cryptogenic stroke was brought about by a recent meta-analysis by Udell et al [17]. This meta-analysis, based on the evaluation of the only three RCTs carried out so far (including a total of 2,303 patients randomized to an invasive approach or conservative strategy), concluded that PFO closure did not significantly reduce the risk of recurrent stroke/TIA (3.7\% vs. 5.2\%; risk ratio ( $\mathrm{RR}$ ): 0.73 ; 95\% $\mathrm{CI}$ : $0.50-1.07 ; \mathrm{P}=0.10$ ).

This meta-analysis also differs from others that have addressed the topic, in that it emphasizes another aspect already summarily highlighted by one of three RCTs [19], yet largely overlooked so far: the sub-optimal safety of transcatheter closure of the PFO, which would be burdened by a significant increase in the risk of arrhythmic destabilization of the atria in the medium term compared with medical therapy (increased risk of incident atrial fibrillation/flutter over a mean follow-up of 2.6 years: $3.8 \%$ vs. $1.0 \%$; RR: 3.67 ; 95\% CI: $1.95-6.89$; $\mathrm{P}<0.0001)$. Another paramount aspect is represented by dis- crepancies found by performing a comparison of the available meta-analyses. Indeed, it is certainly instructive to note (Table 1 [4-18]) that the numerous meta-analyses, which originated from the aggregation of the same data (in any case, 2,303 patients recruited from the same three RCTs) produce conflicting results, depending on the manner adopted for presentation of the pertinent data ("intention to treat", "as treated" or "per protocol", Table 2) as well as on the approach used for evaluation of the effect size ("random effects" versus "fixed effects" model).

Furthermore, it is important, in any case, to remember that none of the three analyzed RCTs, taken individually, had demonstrated a statistically significant favorable effect on the outcome (survival free from stroke or TIA) exerted by the closure of the PFO [26-28]. Then, several meta-analyses recently showed that even by means of pooling data derived from the three RCTs, there was no evidence of statistically significant difference in outcomes by comparing patients assigned to PFO closure with patients left in simple medical therapy $[9,10,13$, $14,17,18]$. In fact, current guidelines from the AHA do not support PFO closure in the event of cryptogenic stroke or TIA unless a deep venous thrombosis is identified [29]. Additionally, closure of a PFO with the use of a percutaneous transcatheter device has been considered so far as an investigational procedure by the Food and Drug Administration (FDA) [19]. Nevertheless, in the USA as well in European Countries, many such patients are treated off-label with devices that are approved for the closure of ostium secundum atrial septal defects $[2,19,26]$.

On the contrary, it should also be noted that other authors have not ruled out at all the possibility of a greater benefit resulting from the closure of the PFO compared with medical treatment $[4,5,7]$ and that there are some who argue the superiority of the interventional option $[11,12,27,28]$. It would 
Table 2. Approaches Used for Assessing a Randomized Controlled Trial: "Intention to Treat", "As Treated" and "Per Protocol"

Intention to treat Randomized clinical trials analyzed by the "intention to treat" (ITT) approach provide unbiased comparisons among the treatment groups. "Intention to treat" analyses are done to avoid the effects of crossover and dropout, which may break the random assignment to the treatment groups in a study. ITT analysis provides information about the potential effects of treatment policy rather than on the potential effects of specific treatment. Since it started in the 1960s, the principle of ITT has become widely accepted for the analysis of controlled clinical trials. However, full application of ITT analysis can only be performed where there are complete outcome data for all randomized subjects.

As treated "As treated" analysis has the general idea of comparing the subjects with the treatment regimen that they received. It does not consider which treatment they were assigned for the treatment.

Per protocol The "per protocol" analysis, also known as an "on-treatment" analysis, can only be restricted to the participants who fulfill the protocol in the terms of the eligibility, interventions, and outcome assessment. Indeed, the "per protocol" analysis restricts the comparison of the treatments to the ideal patients, that is, those who adhered perfectly to the clinical trial instructions as stipulated in the protocol. However, by restricting the analysis to a selected patient population, it does not show the practical value of the drug or method that has to be tested.

also be a useful choice to examine briefly the evidence in favor of PFO closure that has derived from alternative approaches, i.e., the meta-analyses that have considered separately the studies using the "Amplatzer" septal occluder device [20, 21] from the one employing the STARFlex [19] (Fig. 1).

When analyzing these trials, it should be noted that all of the trials were limited by slow recruitment and unexpect- edly low event rates. Higher risk patients were less likely to be randomized, and more likely to receive PFO closure with an off-label device without being enrolled in the RCT. For example, during the recruitment period for the CLOSURE I trial, the utilization of off-label PFO closure versus referral to the randomized trial was $3: 1$, with higher risk patients preferentially being referred to off-label device closure. This ability

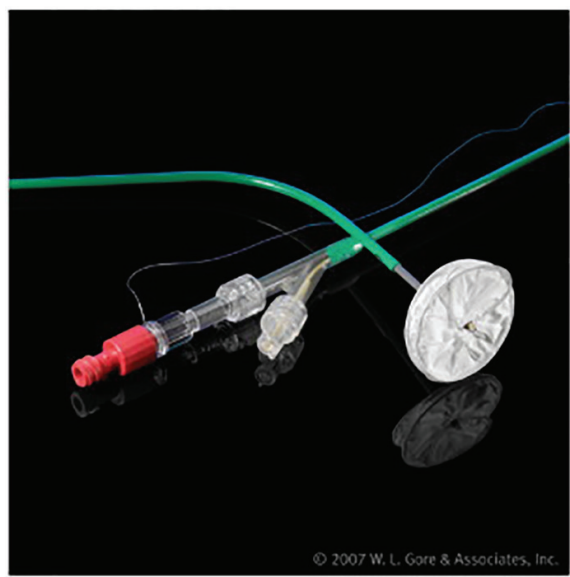

a) Gore Helex Septal Occluder

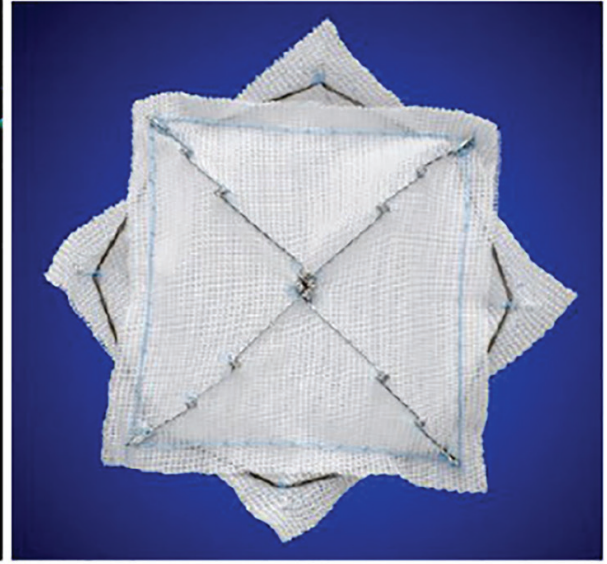

b) STARFlex PFO implant device

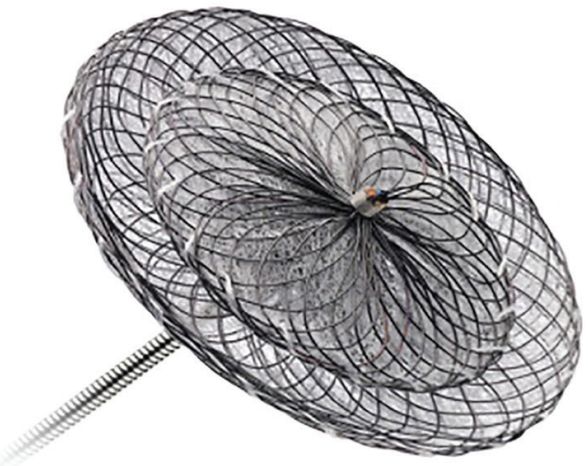

C) Amplatzer PFO Occluder

Figure 1. PFO occluding devices. (a) Gore Helex septal occluder; (b) STARFlex PFO implant device; (c) Amplatzer PFO occluder. 
of patients to obtain PFO closure outside of the trial, with an off-label device meant that the patients who agreed to be randomized tended to have lower risk for recurrence than patients studied in the observational populations, with consequent relatively high probability of lack of significant differences between the outcomes of the two arms through the follow-up.

In addition, the lack of a significant effect on the efficacy endpoint (composite of death, non-fatal stroke, TIA, or peripheral embolism in the PC trial; or composite of all-cause death or ischemic stroke in the RESPECT trial) is no longer confirmed when the analysis is limited to aggregate data derived from trials in which an Amplatzer septal occluder was tested, without incorporating in the meta-analysis the data originating from the CLOSURE I trial, which had instead used the STARFlex device. In this regard, a concordance of several meta-analyses can be found $[4,12,15,16]$; for example, Khan et al [12] show that by pooling the data from the RESPECT trial and PC trial, a borderline protective effect of the Amplatzer device is noticeable against the risk of acute neurological events in individuals with PFO (HR: 0.54; 95\% CI: 0.29 - 1.01).

\section{Future Prospects}

An additional study is underway to investigate the role of PFO closure in secondary prevention of cryptogenic stroke. The REDUCE study is a randomized, multi-center study designed to compare PFO closure using the Gore Helex occluder (Fig. 1) with medical therapy in patients with a history of cryptogenic stroke or imaging confirmed TIA. The trial has completed its recruitment phase and the results are eagerly awaited.

\section{Conclusions}

The practice of closing the PFO did not prove to be helpful in causing a significant reduction in the incidence of recurrent stroke in patients with a history of previous stroke or cryptogenic TIA. Recent evidence has also disclosed that an increased incidence of AF/atrial Fl could involve patients who have undergone transcatheter PFO closure. Thus, while awaiting the results of the upcoming trial on this topic, physicians should remain cautious and adhere to the approach recommended by the guidelines. They attribute some importance to antithrombotic therapy (both by means of anticoagulants and antiplatelet agents), while denying an alleged superior efficacy to transcatheter PFO occlusion, except for in selected cases of patients with documented PFO and a concomitant clinicalinstrumental picture of deep venous thrombosis [29]. Only in the latter, the risk of paradoxical embolism caused by right to left shunt through a PFO may justify the application of a septal occluding device.

\section{Conflict of Interest}

The authors of this article state that they have no conflict of interest to declare concerning the present work.

\section{References}

1. Kistler JP, Furie KL. Patent foramen ovale diameter and embolic stroke: a part of the puzzle? Am J Med. 2000;109(6):506-507.

2. Dalen JE. Are patients with a patent foramen ovale at increased risk of stroke? A billion dollar question. Am J Med. 2007;120(6):472-474.

3. Mojadidi MK, Christia P, Salamon J, Liebelt J, Zaman T, Gevorgyan R, Nezami N, et al. Patent foramen ovale: Unanswered questions. Eur J Intern Med. 2015;26(10):743751.

4. Ntaios G, Papavasileiou V, Makaritsis K, Michel P. PFO closure vs. medical therapy in cryptogenic stroke or transient ischemic attack: a systematic review and meta-analysis. Int J Cardiol. 2013;169(2):101-105.

5. Pineda AM, Nascimento FO, Yang SC, Kirtane AJ, Sommer RJ, Beohar N. A meta-analysis of transcatheter closure of patent foramen ovale versus medical therapy for prevention of recurrent thromboembolic events in patients with cryptogenic cerebrovascular events. Catheter Cardiovasc Interv. 2013;82(6):968-975.

6. Kitsios GD, Thaler DE, Kent DM. Potentially large yet uncertain benefits: a meta-analysis of patent foramen ovale closure trials. Stroke. 2013;44(9):2640-2643.

7. Riaz IB, Dhoble A, Mizyed A, Hsu CH, Husnain M, Lee JZ, Lotun K, et al. Transcatheter patent foramen ovale closure versus medical therapy for cryptogenic stroke: a meta-analysis of randomized clinical trials. BMC Cardiovasc Disord. 2013;13:116.

8. Hakeem A, Marmagkiolis K, Hacioglu Y, Uretsky BF, Gundogdu B, Leesar M, Bailey SR, et al. Safety and efficacy of device closure for patent foramen ovale for secondary prevention of neurological events: Comprehensive systematic review and meta-analysis of randomized controlled trials. Cardiovasc Revasc Med. 2013;14(6):349355.

9. Kwong JS, Lam YY, Yu CM. Percutaneous closure of patent foramen ovale for cryptogenic stroke: a metaanalysis of randomized controlled trials. Int $\mathrm{J}$ Cardiol. 2013;168(4):4132-4138.

10. Nagaraja V, Raval J, Eslick GD, Burgess D, Denniss AR. Is transcatheter closure better than medical therapy for cryptogenic stroke with patent foramen ovale? A meta-analysis of randomised trials. Heart Lung Circ. 2013;22(11):903-909.

11. Rengifo-Moreno P, Palacios IF, Junpaparp P, Witzke CF, Morris DL, Romero-Corral A. Patent foramen ovale transcatheter closure vs. medical therapy on recurrent vascular events: a systematic review and metaanalysis of randomized controlled trials. Eur Heart J. 2013;34(43):3342-3352.

12. Khan AR, Bin Abdulhak AA, Sheikh MA, Khan S, Erwin PJ, Tleyjeh I, Khuder S, et al. Device closure of patent foramen ovale versus medical therapy in cryptogenic stroke: a systematic review and meta-analysis. JACC Cardiovasc Interv. 2013;6(12):1316-1323.

13. Spencer FA, Lopes LC, Kennedy SA, Guyatt G. System- 
atic review of percutaneous closure versus medical therapy in patients with cryptogenic stroke and patent foramen ovale. BMJ Open. 2014;4(3):e004282.

14. Wolfrum M, Froehlich GM, Knapp G, Casaubon LK, DiNicolantonio JJ, Lansky AJ, Meier P. Stroke prevention by percutaneous closure of patent foramen ovale: a systematic review and meta-analysis. Heart. 2014;100(5):389395.

15. Capodanno D, Milazzo G, Vitale L, Di Stefano D, Di Salvo M, Grasso C, Tamburino C. Updating the evidence on patent foramen ovale closure versus medical therapy in patients with cryptogenic stroke: a systematic review and comprehensive meta-analysis of 2,303 patients from three randomised trials and 2,231 patients from 11 observational studies. EuroIntervention. 2014;9(11):1342-1349.

16. Pandit A, Aryal MR, Pandit AA, Jalota L, Kantharajpur S, Hakim FA, Lee HR. Amplatzer PFO occluder device may prevent recurrent stroke in patients with patent foramen ovale and cryptogenic stroke: a meta-analysis of randomised trials. Heart Lung Circ. 2014;23(4):303-308.

17. Udell JA, Opotowsky AR, Khairy P, Silversides CK, Gladstone DJ, O'Gara PT, Landzberg MJ. Patent foramen ovale closure vs medical therapy for stroke prevention: meta-analysis of randomized trials and review of heterogeneity in meta-analyses. Can J Cardiol. 2014;30(10):1216-1224.

18. Li J, Liu J, Liu M, Zhang S, Hao Z, Zhang J, Zhang C. Closure versus medical therapy for preventing recurrent stroke in patients with patent foramen ovale and a history of cryptogenic stroke or transient ischemic attack. Cochrane Database Syst Rev. 2015;9:CD009938.

19. Furlan AJ, Reisman M, Massaro J, Mauri L, Adams H, Albers GW, Felberg R, et al. Closure or medical therapy for cryptogenic stroke with patent foramen ovale. N Engl J Med. 2012;366(11):991-999.

20. Meier B, Kalesan B, Mattle HP, Khattab AA, Hildick-
Smith D, Dudek D, Andersen G, et al. Percutaneous closure of patent foramen ovale in cryptogenic embolism. N Engl J Med. 2013;368(12):1083-1091.

21. Carroll JD, Saver JL, Thaler DE, Smalling RW, Berry S, MacDonald LA, Marks DS, et al. Closure of patent foramen ovale versus medical therapy after cryptogenic stroke. N Engl J Med. 2013;368(12):1092-1100.

22. Hara H, Virmani R, Ladich E, Mackey-Bojack S, Titus J, Reisman M, Gray W, et al. Patent foramen ovale: current pathology, pathophysiology, and clinical status. J Am Coll Cardiol. 2005;46(9):1768-1776.

23. Hagen PT, Scholz DG, Edwards WD. Incidence and size of patent foramen ovale during the first 10 decades of life: an autopsy study of 965 normal hearts. Mayo Clin Proc. 1984;59(1):17-20.

24. Homma S, Sacco RL. Patent foramen ovale and stroke. Circulation. 2005;112(7):1063-1072.

25. Hausmann D, Mugge A, Becht I, Daniel WG. Diagnosis of patent foramen ovale by transesophageal echocardiography and association with cerebral and peripheral embolic events. Am J Cardiol. 1992;70(6):668-672.

26. Maisel WH, Laskey WK. Patent foramen ovale closure devices: moving beyond equipoise. JAMA. 2005;294(3):366-369.

27. Tobis J. Patent foramen ovale and the risk of cryptogenic stroke. Cleve Clin J Med. 2014;81(7):425-426.

28. Schuchlenz HW, Weihs W, Horner S, Quehenberger F. The association between the diameter of a patent foramen ovale and the risk of embolic cerebrovascular events. Am J Med. 2000;109(6):456-462.

29. Kernan WN, Ovbiagele B, Black HR, Bravata DM, Chimowitz MI, Ezekowitz MD, Fang MC, et al. Guidelines for the prevention of stroke in patients with stroke and transient ischemic attack: a guideline for healthcare professionals from the American Heart Association/American Stroke Association. Stroke. 2014;45(7):2160-2236. 